\title{
ATIVIDADE FÍSICA AO AR LIVRE E A INFLUÊNCIA NA QUALIDADE DE VIDA
}

Daniel Fernando Dos Reis, Francione Da Silva Souza, Juliana Da Silva Jesus, Thiago Alves Garcia, Guilherme Akio Tamura Ozaki, Everton Alex Carvalho Zanuto, Adriana Junqueira, Robson Chacon Castoldi, Regina Celi Trindade Camargo, José Carlos Silva Camargo Filho

Universidade do Oeste Paulista - UNOESTE, Faculdade de Educação Física, Presidente Prudente, SP. E-mail: fernando.daniel28@yahoo.com.br.

\section{RESUMO}

Áreas públicas são consideradas de fácil acesso e representam uma alternativa de ampliação de locais favoráveis à prática de atividades físicas e melhora da qualidade de vida. O objetivo do presente estudo foi investigar a influência da prática de atividades físicas ao ar livre na qualidade de vida de seus praticantes. Para isso, foi realizada uma revisão de literatura por meio das bases de dados eletrônicas "Google Acadêmico", "Lilacs" e "Scielo". A busca foi delimitada ao período de 2008 a 2017. Foram selecionados 21 artigos para a elaboração do estudo. Foi verificado que a atividade física ao ar livre pode ser considerada como uma estratégia de promoção à saúde e proporciona benefícios de ordem física, social e cultural. Conclui-se que, praticar atividades físicas ao ar livre evita o sedentarismo e outros agraves à saúde, considerados sérios problemas para a saúde física e mental nos dias atuais.

Palavras chave: Atividade física, Estudos de revisão, Parques públicos, Qualidade de vida, Saúde.

\section{PHYSICAL ACTIVITY OUTDOORS AND THE INFLUENCE ON QUALITY OF LIFE}

\begin{abstract}
Public areas are considered to be easily accessible and represent an alternative to expansion of places conducive to the practice of physical activities and improving the quality of life. The objective of this study was investigate the influence that practice of outdoor physical activities on quality of life in yours practitioners. We performed a literature review through the electronic databases Google Scholar, Lilacs and Scielo. The search specified in the period 2008 to 2017. We selected 21 articles for the elaboration of the study. The use of such public structures should be considered as a strategy of health promotion that provides benefits from the physical, social and cultural development, improving the quality of life of its users through the implementation of outdoor physical activities.
\end{abstract}

Keywords: Physical activity, Studies of revision, Public Parks, Quality of life, Health. 


\section{INTRODUÇÃO}

A atividade física é classificada como um fator de proteção e promoção da saúde e esta relacionada a inúmeros benefícios como a redução de peso, melhora cardiovascular, redução de doenças crônicas, diminuição do risco de morte prematura, entre outros ${ }^{1}$. E infelizmente existe alta prevalência de inatividade física em uma para cada cinco pessoas ao redor do mundo. Além disso, é predominante em países urbanizados, dos quais ocorre principalmente em idosos e mulheres ${ }^{1}$.

Nesse sentido, realizar atividades físicas regularmente é importante para restabelecer a saúde diante dos danos que a rotina estressante da atualidade causa nos indivíduos, sendo relevante aos três níveis de atenção à saúde, o primário, secundário e terciário ${ }^{2}$.

Com o crescente aumento da construção de parques e praças públicas nas cidades, vem se valorizando a cada dia a utilização destes espaços para a prática de atividades físicas pela população em geral. Uma vez que esses locais são de fácil acesso e classificados como ambientes sociais de lazer e de manutenção da saúde ${ }^{3}$.

Usufruir dos espaços públicos para a realização de atividades físicas faz com que se eleve a qualidade de vida da população, previna doenças, e aumente a socialização e a consciência ambiental dos indivíduos ${ }^{4}$. A prática de atividades físicas em áreas verdes e espaços públicos proporcionam a seus praticantes benefícios imediatos como normalização dos níveis de adrenalina e noradrenalina, dos níveis de glicose sanguínea e melhora na qualidade do sono; e a longo prazo como a diminuição da incidência de doenças músculos-esqueléticos, cardiovasculares e metabólicas 5 .

De acordo com Polisseni e Ribeiro ${ }^{1}$, a atividade física deve ser incentivada por meio de iniciativas tanto do poder público quanto do privado. É preciso levar em consideração as particularidades dos indivíduos, sejam elas financeiras e/ou sociais, disponibilizando alternativas acessíveis para a prática de atividades físicas ao ar livre, visando atingir coletivamente a busca por um estilo de vida mais saudável.

Justifica-se o presente estudo pela crescente procura de atividades físicas ao ar livre, que são importantes ferramentas para a promoção da saúde e qualidade de vida. Dessa forma, o objetivo d presente estudo foi investigar a influência da prática de atividades físicas ao ar livre na qualidade de vida de seus praticantes.

\section{METODOLOGIA}

Trata-se de um estudo de revisão de literatura. Na revisão de literatura se avalia a produção bibliográfica de um tema específico em um determinado período de anos, apresentando-se novas ideias da literatura selecionada somando conhecimentos. A pesquisa bibliográfica é realizada coletando e armazenando os dados obtidos através dos artigos selecionados sobre o assunto em questão, seguido da leitura e fichamentos das informações importantes para a elaboração da revisão de literatura ${ }^{6}$.

Os critérios de inclusão para a elaboração do presente estudo foram: artigos publicados em português, no período de 2008 a 2017, que abordavam sobre atividade física ao ar livre e a influencia na qualidade de vida. Os artigos utilizados estavam registrados nas bases de dados Google Acadêmico, Lilacs (Literatura Latino-Americana e do Caribe em Ciências da Saúde) e Scielo (Scientific Electronic Library Online). Foram utilizadas as palavras-chaves "atividades de lazer" e "atividade física" e "estudos de revisão" e "parques públicos" e "qualidade de vida" e "saúde".

Foram selecionados 21 artigos que abordavam o tema proposto. Os artigos selecionados foram agrupados em quatro categorias: a) atividades físicas e exercícios físicos; b) atividades físicas ao ar livre; c) influência da atividade física ao ar livre na qualidade de vida; d) relevância do educador físico nas atividades ao ar livre. 


\section{RESULTADOS E DISCUSSÃO ATIVIDADES FíSICAS E EXERCíCIOS FÍSICOS}

Foi verificado na tabela 1 que um estilo de vida saudável depende de bons hábitos alimentares e pratica de atividades físicas junto a uma boa saúde mental.

Tabela 1. Atividades físicas e exercícios físicos

\begin{tabular}{|c|c|c|}
\hline Autor & Ano & Abordagem \\
\hline MACIEL $^{7}$ & 2010 & $\begin{array}{l}\text { Manter-se um estilo de vida saudável é preciso que a rotina diária } \\
\text { de um indivíduo apresente bons hábitos alimentares e prática } \\
\text { regular de atividades físicas, reduzindo as consequências à saúde } \\
\text { causadas pela Inatividade Física. }\end{array}$ \\
\hline SILVA et al. ${ }^{8}$ & 2010 & $\begin{array}{l}\text { O nível de atividade física de uma pessoa esta a qualidade de vida } \\
\text { da mesma, não apenas relacionado aos aspectos de saúde física, } \\
\text { mas também aos aspetos psicológicos e cognitivos, } \\
\text { independentemente do sexo, idade e profissão deste indivíduo. }\end{array}$ \\
\hline $\begin{array}{l}\text { SANTA CLARA } \\
\text { et al. }{ }^{9}\end{array}$ & 2015 & $\begin{array}{l}\text { A atividade física é compreendida como qualquer movimento } \\
\text { corporal realizado pela musculatura que leve a um gasto de } \\
\text { energia maior do que o indivíduo apresenta quando se encontra } \\
\text { em repouso. }\end{array}$ \\
\hline $\begin{array}{l}\text { SANTA CLARA } \\
\text { et al. }{ }^{9}\end{array}$ & 2015 & $\begin{array}{l}\text { Os exercícios físicos são definidos como toda atividade física } \\
\text { organizada, elaborada e contínua que visa melhorar e manter a } \\
\text { função cardiorrespiratória, a composição corporal, flexibilidade, } \\
\text { força e resistência. }\end{array}$ \\
\hline MACIEL $^{7}$ & 2010 & $\begin{array}{l}\text { Os exercícios físicos estão relacionados à performance, direcionado } \\
\text { à melhora do desempenho esportivo. }\end{array}$ \\
\hline MACIEL $^{7}$ & 2010 & $\begin{array}{l}\text { A Inatividade Física nos dias atuais é resultado do crescente avanço } \\
\text { tecnológico que trouxe mais conforto à vida das pessoas, } \\
\text { reduzindo o incentivo à movimentação devido às diversas opções } \\
\text { de lazer presente em suas casas. }\end{array}$ \\
\hline
\end{tabular}

Manter-se saudável nos dias atuais não tem se tornado uma tarefa fácil, frente a todas as comodidades proporcionadas pelos avanços tecnológicos. Para manter-se um estilo de vida saudável é preciso que a rotina diária de um indivíduo apresente bons hábitos alimentares e prática regular de atividades físicas, compreendendo a relevância que a prática destas atividades tem na promoção da saúde e na redução dos fatores de risco ${ }^{7}$.

Proporcional ao nível de atividade física de uma pessoa esta a qualidade de vida da mesma, não apenas relacionado aos aspectos de saúde física, mas também aos aspetos psicológicos e cognitivos, independentemente do sexo, idade e profissão deste indivíduo ${ }^{8}$. 0 conceito de atividade física é compreendido como qualquer movimento corporal realizado pela musculatura que leve a um gasto de energia maior do que o indivíduo apresenta quando se encontra em repouso, como na caminhada, dança, cuidar do jardim, entre outros ${ }^{9}$.

Já os exercícios físicos são definidos como toda atividade física organizada, elaborada e contínua que visa melhorar e manter a função cardiorrespiratória, a composição corporal, a flexibilidade, a força e resistência muscular ${ }^{9}$. Para $\mathrm{Maciel}^{7}$, a atividade física esta direcionada a melhorar a qualidade de vida do praticante, reduzindo as consequências à saúde causadas pelo sedentarismo. 0 exercícios físicos segundo o autor estão relacionados à performance, direcionado à melhora do desempenho esportivo. 
Um grande risco à saúde na atualidade é a inatividade física. Um indivíduo considerado inativo fisicamente é aquele que realiza em sua rotina diária um mínimo de atividade física, apresentando um gasto energético semanal de $500 \mathrm{kcal}$ provenientes da locomoção, trabalho, lazer e atividades domésticas ${ }^{7}$.

De acordo com Maciel $^{7}$, a inatividade física nos dias atuais é resultado do crescente avanço tecnológico que trouxe mais conforto à vida das pessoas, reduzindo o incentivo à movimentação devido às diversas opções de lazer presente em suas casas como a televisão, computador, vídeo games, entre outros; assim como pela insegurança urbana e insuficiência de espaços que possibilitam a prática atividades físicas ao ar livre.

A prática de atividades físicas é considerada um meio de lazer que visa reduzir o estresse presente na rotina diária dos indivíduos, sendo uma atividade agradável e viciante que reduz sensações desconfortáveis como a ansiedade, irritabilidade e depressão, além de manter uma boa forma física e melhora da qualidade de vida ${ }^{8}$.

Psicologicamente a prática de atividades físicas proporciona benefícios sobre a autoimagem e autoestima do praticante, fisicamente está relacionada à diminuição de doenças cardiovasculares, de mortes prematuras, redução dos casos de acidente vascular cerebral, câncer de mama, câncer cólon, e de diabetes mellitus tipo II .

Esta prática também esta relacionada à prevenção e redução da obesidade, da hipertensão arterial, da osteoporose, do estresse, da ansiedade e da depressão promovendo bem-estar aos seus adeptos ${ }^{7}$. De acordo com Júnior ${ }^{10}$, a prática regular de atividade física promove inúmeros benefícios a todas as faixas etárias, melhorando a capacidade funcional e habilidade física destes indivíduos, promovendo a socialização, a longevidade, melhorando o estado mental e níveis de humor.

\section{ATIVIDADES FÍSICAS AO AR LIVRE}

Observa-se na tabela 2 que a busca pela atividade ao ar livre tem aumentado devido a acessibilidade, beleza, a socialização e combinado com atividade física em viárias intensidades. 
Tabela 2. Atividades físicas ao ar livre

\begin{tabular}{lll}
\hline Autor & Ano & Abordagem \\
\hline SOUZA et al. $^{3}$ & 2017 & $\begin{array}{l}\text { É crescente na atualidade a conscientização da população sobre a } \\
\text { importância da utilização de espaços públicos ao ar livre para a prática } \\
\text { de atividades físicas e lazer, promovendo a convivência social e a } \\
\text { preservação ambiental nos grandes centros urbanos }\end{array}$ \\
\hline $\begin{array}{l}\text { PIERONE et } \\
\text { al. }^{4}\end{array}$ & $2016 \begin{array}{l}\text { A beleza natural dos parques públicos, estes locais se tornaram pontos } \\
\text { de bem-estar e saúde, sendo fundamentais para a prevenção de } \\
\text { doenças e agravos à saúde, que associado à prática de atividades físicas } \\
\text { geram benefícios sociais, físicos e psicológicos. }\end{array}$ \\
\hline $\begin{array}{l}\text { KUNZLER et } \\
\text { al. }^{2}\end{array}$ & $2014 \begin{array}{l}\text { Nestes espaços é possível observar uma grande variedade de tipos de } \\
\text { atividades realizadas em diferentes níveis de intensidades e em } \\
\text { diferentes faixas etárias }\end{array}$ \\
\hline
\end{tabular}

SILVA et al. ${ }^{11} 2015$ Ter espaços públicos acessíveis, importante que estes espaços ofereçam segurança aos usuários e que estejam em constante manutenção para que possibilitem a pratica de atividades físicas e atividades de lazer sem nenhum transtorno às pessoas adeptas a este estilo de vida saudável em meio à natureza e meio ambiente.

COLLET et al. 2008 O que leva as pessoas a praticarem atividades ao ar livre são estímulos 12 por parte de familiares, amigos ou colegas de trabalho por meio da influência social e da busca por um estilo de vida mais saudável.

CASTAÑON et 2016 São espaços que permitem que diferentes faixas etárias estejam em um al. ${ }^{13}$ mesmo espaço buscando momentos de lazer ou de condicionamento físico, melhorando a qualidade de vida e de saúde desta população.

Alguns fatores são determinantes para a realização de atividades físicas pela população como influências sociais, políticas, culturais e econômicas, refletindo diretamente no nível de saúde destes indivíduos. É crescente na atualidade a conscientização da população sobre a importância da utilização de espaços públicos ao ar livre para a prática de atividades físicas e lazer, promovendo a convivência social e a preservação ambiental nos grandes centros urbanos ${ }^{3}$.

Devido à acessibilidade e a beleza natural dos parques públicos, estes locais se tornaram pontos de bem-estar e saúde, sendo fundamentais para a prevenção de doenças e agravos à saúde, além de proporcionarem lazer e diversão às pessoas que os usufruem ${ }^{4}$ (PIERONE et al, 2016). A implantação deste tipo de espaço próximo a áreas residenciais proporciona aos moradores locais e próximos uma área de promoção da saúde, favorecendo um estilo de vida mais ativo e saudável para esta população ${ }^{3}$.

Os parques públicos vêm se tornando uma referência em saúde, pois possibilitam a interação entre homem e meio ambiente, que associado à prática de atividades físicas geram benefícios sociais, físicos e psicológicos ${ }^{4}$. A prática de atividades físicas nestes espaços trazem pontos positivos aos praticantes, pois sem nenhum custo e com fácil acesso melhoram a qualidade de vida da população que reside próximo a estes locais, estimulando a socialização entre os usuários dos espaços e promovendo a sobrevivência ecológica nos centros urbanos ${ }^{3}$.

Praças, áreas verdes e ciclovias são exemplos de espaços que possuem um papel importante na prática de atividades físicas ao ar livre, promovendo um estilo de vida mais ativo à população urbana ${ }^{11}$. Nestes espaços é possível observar uma grande variedade de tipos de atividades realizadas em diferentes níveis de intensidades e em diferentes faixas etárias². 
Dentre as atividades físicas mais praticadas pelas pessoas nos espaços públicos ao ar livre está a caminhada, a corrida, treinamento funcional, vôlei de areia, futebol, andar de skate, entre outras $^{3}$. A caminhada que é um exemplo de atividade física acessível que pode ser realizada em espaços públicos e áreas verdes, esta atividade não tem a necessidade do uso de equipamentos especiais e através dela é possível estimular a população a se tornar fisicamente mais ativa ${ }^{2}$.

Segundo Silva et al. ${ }^{11}$, ter espaços públicos acessíveis e próximos às residências das pessoas não é o suficiente para a adesão das mesmas ao uso destes espaços, é preciso que os mesmos tenham condições adequadas e seguras para o seu uso, para que sejam lugares promotores de saúde e lazer. Nesse sentido é de suma importância que estes espaços ofereçam segurança aos usuários e que estejam em constante manutenção para que possibilitem a pratica de atividades físicas e atividades de lazer sem nenhum transtorno às pessoas adeptas a este estilo de vida saudável em meio à natureza e meio ambiente ${ }^{11}$.

De acordo com Silva et al. ${ }^{11}$, é importante que nestes locais não existam vidros quebrados, indício do uso de álcool e drogas ilícitas, animais soltos, sujeira de animais e lixo, sinais de vandalismo ou má manutenção da grama. É preciso também que possuam boa iluminação para a segurança de seus usuários, banheiros públicos em boa condição para comodidade e higiene, assim como bancos para o conforto e momentos de descanso após a prática de atividades físicas e lazer ${ }^{11}$.

O que leva as pessoas a praticarem atividades ao ar livre são estímulos por parte de familiares, amigos ou colegas de trabalho que já são adeptos a esta pratica, ocorrendo desta forma por meio da influência social e da busca por um estilo de vida mais saudável ${ }^{12}$. Estes espaços são como pontos de encontros que proporcionam um momento de lazer e estimulam a prática de atividades físicas e de esportes pela população em geral, visto que não são todas as pessoas que conseguem pagar ou se deslocar até uma academia particular. São espaços que permitem que diferentes faixas etárias estejam em um mesmo espaço buscando momentos de lazer ou de condicionamento físico, melhorando a qualidade de vida e de saúde desta população ${ }^{16}$.

\section{INFLUÊNCIA DA ATIVIDADE AO AR LIVRE NA QUALIDADE DE VIDA}

Observando a Tabela 3 vemos que a atividade física ao ar livre promove relaxamento mental proporcionado pela natureza pela socialização recreativas. 
Tabela3. Influência da atividade ao ar livre na qualidade de vida

\begin{tabular}{|c|c|c|}
\hline Autor & Ano & Abordagem \\
\hline $\begin{array}{l}\text { VASCONCELOS; } \\
\text { ROSA }^{14}\end{array}$ & 2016 & $\begin{array}{l}\text { Estes espaços são cada vez mais procurados para a prática de } \\
\text { atividades físicas ou apenas para o relaxamento mental, criando a } \\
\text { sensação de paz e tranquilidade proporcionada pela simples } \\
\text { contemplação da natureza. }\end{array}$ \\
\hline LONDE et al. $^{5}$ & 2014 & $\begin{array}{l}\text { Indicadores de qualidade de vida da população urbana, pois estão } \\
\text { relacionados ao nível de lazer, recreação e atualmente de atividade } \\
\text { física destes indivíduos, favorecendo a socialização e demonstrando } \\
\text { o nível de vida comunitária desta população, é importante uma boa } \\
\text { limpeza, acessibilidade, presença de áreas verdes e espaços } \\
\text { públicos. }\end{array}$ \\
\hline $\begin{array}{l}\text { SZEREMETA; } \\
\text { ZANNIN }^{15}\end{array}$ & 2013 & $\begin{array}{l}\text { A motivação das pessoas para usarem parques públicos como fonte } \\
\text { de lazer e de realização de atividades físicas proporcionada pela } \\
\text { vegetação arbórea destes locais, que reduz o cansaço mental } \\
\text { ocasionado pela estressante rotina das cidades. }\end{array}$ \\
\hline SILVA et al. ${ }^{11}$ & 2015 & $\begin{array}{l}\text { É importante a preservação dos espaços públicos e continuidade da } \\
\text { elaboração de projetos que visem a construção de áreas verdes, são } \\
\text { alternativas que visam melhorar a qualidade de vida das pessoas } \\
\text { que vivem em centros urbanos, satisfazendo suas necessidades } \\
\text { físicas, sociais e psicológicas através da influencia do meio ambiente. }\end{array}$ \\
\hline
\end{tabular}

A busca do ser humano pela qualidade de vida através de um estilo de vida mais saudável, nunca esteve tão em foco como nos dias atuais. A escolha por manter um estilo de vida mais saudável é um dos grandes objetivos das pessoas nos grandes centros urbanos. Parques e áreas verdes tem o objetivo de reduzir as consequencias de negativas resultantes da acelerada e desenfreada urbanização, fazendo com que as pessoas procurem estes locais para a prática de atividades físicas, para o relaxamento mental, ou apenas para a simples contemplação da natureza ${ }^{14}$.

Parques públicos são considerados uma área verde urbana constituída predominantemente de vegetação e alguns dos elementos formadores do meio ambiente, apresentando diferentes finalidades dentro da cidade, como lazer, estética e ecologia ${ }^{15}$.

São importantes indicadores de qualidade de vida da população urbana pois estão relacionados ao nível de lazer e recreação destes indivíduos, sendo conseiderados locais de socialização que demonstrando o nível de vida comunitária desta população ${ }^{5}$.

Para Silva et al. ${ }^{11}$, atualmente o entendimento pelas pessoas a respeito da qualidade de vida é estabelecida a partir de dois parâmetros: bem estar, felicidade, amor, prazer e realização pessoal.

A qualidade de vida de uma população é medida de acordo com o grau de satisfação de suas necessidades básicas, através da disponibilidade de serviços básicos. Para que se tenha uma qualidade de vida urbana é importante uma boa iluminação, limpeza, acessibilidade, qualidade de moradia, presença de áreas verdes e espaços públicos, entre outros indicadores ${ }^{5}$.

Os espaços públicos com áreas verdes minimizam as características negativas presentes nos centros urbanos como a poluição do ar, sonora e visual produzidos nestes locais, fazendo com que as pessoas retornem à natureza e se beneficiem com esse contato.

Estes locais além de reduzirem as poluições existentes nos centros urbanos proporcionam conforto térmico devido à vegetação arbórea, elevando a qualidade de vida de seus frequentadores ${ }^{5}$. 
A motivação das pessoas para usarem parques públicos como fonte de lazer e de realização de atividades físicas esta diretamente ligada à sensação proporcionada pela vegetação arbórea destes locais, que reduz o esgotamento e cansaço mental ocasionado pela estressante rotina das cidades, reduzindo também o nível de sedentarismo destes indivíduos ${ }^{15}$.

Para se ter ideia, com apenas cinco minutos de caminhada nestes locais é possível se reduzir os impactos na saúde mental gerados pelo estresse presente nos centros urbanos, melhorando o humor e a auto estima de seus praticantes. Observa-se também a prática de atividades físicas em áreas verdes promovem a consciência ambiental da população, mostrando a importância destes espaços para a qualidade de vida destes indivíduos ${ }^{15}$.

A prática de atividades físicas ao ar livre proporciona inúmeros benefícios à saúde de seus praticantes. Em curto prazo ocorre uma normalização da glicose sanguínea, adrenalina e noradrenalina; assim como a melhora na qualidade de sono. Em longo prazo ocorre melhora da função cardiovascular; das habilidades cognitivas; redução do aparecimento de doenças músculosesqueléticas, cardiovasculares e metabólicas ${ }^{5}$.

É importante a preservação dos espaços públicos e continuidade da elaboração de projetos que visem a construção de áreas verdes para convívio social. De forma que possa ampliar as alternativas que visam melhorar a qualidade de vida das pessoas que vivem em centros urbanos, satisfazendo suas necessidades físicas, sociais e psicológicas através da influencia do meio ambiente ${ }^{16}$.

Relevância do educador físico nas atividades ao ar livre.

Tabela 4. Relevância do educador físico nas atividades ao ar livre.

\begin{tabular}{|c|c|c|}
\hline Autor & Ano & Abordagem \\
\hline $\begin{array}{l}\text { SILVA; PETROSKI; } \\
\text { REIS }^{17}\end{array}$ & 2009 & $\begin{array}{l}\text { A prática de atividades físicas ao ar livre vem sendo realizadas com } \\
\text { uma grande frequência nos dias atuais, desde atividades de baixo } \\
\text { gasto calórico a atividades exaustivas como corridas de resistência, } \\
\text { importante promotor da saúde. }\end{array}$ \\
\hline SILVA $^{18}$ & 2014 & $\begin{array}{l}\text { O educador físico desempenha o papel de direcionar a prática de } \\
\text { atividades físicas, exercícios físicos e esportes de alto rendimento, } \\
\text { atuando no contexto da educação formal e não formal (no lazer). }\end{array}$ \\
\hline SCABAR et al. ${ }^{19}$ & 2012 & $\begin{array}{l}\text { A relevância do educador físico para a população esta em prevenir, } \\
\text { promover, proteger e reabilitar a saúde destes indivíduos através da } \\
\text { aquisição de hábitos saudáveis, como o incentivo à prática de } \\
\text { atividades físicas regulares. }\end{array}$ \\
\hline GIOLO et al. ${ }^{20}$ & 2016 & $\begin{array}{l}\text { A realização de atividades físicas orientadas por um profissional } \\
\text { capacitado interfere de uma maneira positiva na saúde dos } \\
\text { indivíduos, melhorando a qualidade de vida de uma maneira segura } \\
\text { e positiva }\end{array}$ \\
\hline KUNZLER et al. ${ }^{2}$ & 2014 & $\begin{array}{l}\text { É importante para avaliar e direcionar a atividade física mais } \\
\text { apropriada para cada objetivo e condição de saúde do praticante, } \\
\text { evitando consequências como lesões musculoesqueléticas. }\end{array}$ \\
\hline ARANA; XAVIER ${ }^{21}$ & 2016 & $\begin{array}{l}\text { Ainda existem muitas pessoas que não frequentam parques públicos } \\
\text { para a prática de atividades físicas pela falta de orientação de um } \\
\text { profissional de educação física. }\end{array}$ \\
\hline
\end{tabular}


A prática de atividades físicas ao ar livre vem sendo realizadas com uma grande frequência nos dias atuais, desde atividades de baixo gasto calórico a atividades exaustivas como corridas de resistência. Esta realidade fez com que diferentes campos da saúde como a saúde pública, arquitetura, urbanismo, recreação e psicologia se unissem para apoiar essa prática em espaços públicos de convívio social, reconhecendo ser um importante promotor da saúde ${ }^{17}$.

O educador físico desempenha o papel de direcionar a prática de atividades físicas, exercícios físicos e esportes de alto rendimento, atuando no contexto da educação formal e não formal (no lazer) $)^{18}$.

A relevância do educador físico para a população esta em prevenir, promover, proteger e reabilitar a saúde destes indivíduos através da aquisição de hábitos saudáveis, como o incentivo à prática de atividades físicas regulares ${ }^{19}$.

A realização de atividades físicas orientadas por um profissional capacitado interfere de uma maneira positiva na saúde dos indivíduos, melhorando a qualidade de vida de uma maneira segura e positiva ${ }^{20}$. Este profissional é importante para avaliar e direcionar a atividade física mais apropriada para cada objetivo e condição de saúde do praticante, evitando consequências como lesões musculoesqueléticas ${ }^{2}$.

Praticas de atividades físicas ao ar livre, orientadas por educadores físicos melhora indicadores de saúde e previne doenças, sendo fundamentais para a redução dos níveis de agravos a saúde nos centros urbanos ${ }^{20}$. Entretanto, ainda existem muitas pessoas que não frequentam parques públicos para a prática de atividades físicas pela falta de orientação de um profissional de educação física $^{21}$.

É fundamental que parta destes profissionais a motivação e instrução para uma prática segura e eficaz visando à melhora da qualidade de vida e saúde desta população. Deve partir do educador físico o papel de conscientizar as pessoas de que não apenas espaços fechados e estruturados como academias são locais propícios para a prática de atividades físicas, mas também parques públicos, que em muitos casos possuem uma estrutura física ideal para se praticar desde uma simples caminhada até exercícios intensos voltados para a estética e melhora da saúde em geral ${ }^{21}$.

Este profissional é primordial para direcionar e estimular os indivíduos a se tornarem fisicamente ativos e consequentemente mais saudáveis, por meio da conscientização de que com a mudança de seus comportamentos e do estilo de vida é possível transformar suas vidas para melhor ${ }^{19}$.

\section{CONCLUSÃO}

Conclui-se que praticar atividades físicas ao ar livre evita o sedentarismo e de outros agraves à saúde, considerados sérios problemas para a saúde mental e física nos dias atuais. 0 educador físico tem um papel fundamental no incentivo desta prática visando à promoção da saúde da população em geral, realizando prescrições e orientações adequadas à individualidade de cada aluno, evitando possíveis lesões.

Observa-se também que, a falta de políticas públicas relacionadas ao incentivo à pratica de atividades físicas orientadas em parques públicos, pode estar relacionada a questões esportivas, políticas, culturais e sociais, de municípios que não promovem esta ação.

\section{REFERÊNCIAS}

1. Polisseni, M. L. C.; Ribeiro, L. C.. Exercício físico como fator de proteção para a saúde em servidores públicos. Revista Brasileira de Medicina do Esporte, 2014;20(5):340-344,. Disponível em:

$86922014000500340 \&$ script=sci_abstract $\&$ tIng=es. 
2. Kunzler, M. R. Rocha ES, Bombach GD, Neves D, Santos GS, Carpes FP. Saúde no parque: características de praticantes de caminhada em espaços públicos de lazer. Saúde debate, 2014; 38 (102): 646-653. DOI: http://dx.doi.org/10.5935/0103-1104.20140060,

3. SOUZA, R. G. et al. A influência da prática da atividade Física ao ar livre no desenvolvimento social de Capitais do nordeste. Caderno de Graduação-Ciências Biológicas e da Saúde-UNIT. 2017;4(1):77.

Disponível

em:

https://periodicos.set.edu.br/index.php/cadernobiologicas/article/view/3647.

4. Pierone JM, Vizzotto MM, Heleno MGV, Farhat CAV, Serafim AP. Qualidade de vida de usuários de parques públicos. Bol. Psicol. São Paulo. 2016;66(144):99-112. Disponível em: http://pepsic.bvsalud.org/scielo.php?script=sci arttext\&pid=S0006-

$\underline{59432016000100009 \& \operatorname{lng}=p t \& n r m=i s o}$

5. Londe PR, Mendes PC. A influência das áreas verdes na qualidade de vida urbana. Hygeia: Revista Brasileira de Geografia Médica e da Saúde. 2014; 10 (18): 264. Disponível em: http://www.seer.ufu.br/index.php/hygeia/article/view/26487.

6. Moreira, W. Revisão de Literatura e Desenvolvimento Científico: conceitos e estratégias para confecção. Janus. 2008;1(1):21-30.

Disponível

em:

http://publicacoes.fatea.br/index.php/janus/article/viewArticle/1

7. Maciel MG. Atividade física e funcionalidade do idoso. Motriz. 2010; 16 (4): 1024-32. Disponível em: http://www.scielo.br/pdf/motriz/v16n4/a23v16n4.

8. Silva, RS, Silva I, Silva RA, Souza L, Tomasi E. Atividade física e qualidade de vida. 2010 15(1):115120.

9. Santa-Clara H, Pinto I, Santos V, Pinto R, Melo X, Almeida JP, et al. Atividade física e exercício físico: especificidades no doente cardíaco. Revista Factores de Risco. 2015;35:28-35. Disponível em: http://hdl.handle.net/10400.17/2154.

10. JÚNIOR FFG, Brandão $A B$, Almeida FJM, Oliveira JGD. Compreensão de Idosos sobre os Benefícios da Atividade Física. Revista Brasileira de Ciências da Saúde. 2015;19(30):193-198. DOI: http://dx.doi.org/10.4034/RBCS.2015.19.03.04.

Disponível

em: http://dms.ufpel.edu.br/ares/bitstream/handle/123456789/289/Atividade\%20f\%C3\%ADsica\%20e \%20qualidade\%20de\%20vida.pdf?sequence=1, https://doi.org/10.4034/RBCS.2015.19.03.04.

11. Silva I. Mielke G, Nunes B, Böhm A, Blanke A, Nachtigall M, et al. Espaços públicos de lazer: distribuição, qualidade e adequação à prática de atividade física. Revista Brasileira de Atividade Física \& Saúde. 2015;20(1):82. DOI: http://dx.doi.org/10.12820/rbafs.v.20n1p82.

12. Collet C, Chiaradia BM, Reis RS, Nascimento JV,. Fatores determinantes para a realização de atividades físicas em parque urbano de Florianópolis. Revista Brasileira de Atividade Física \& Saúde. 2008;13(1):15-23. Disponível em: https://periodicos.ufpel.edu.br/ojs2/index.php/RBAFS/article/view/783/792. 
13 Silva EAPC, Silva PPC, Santos ARM, Cartaxo HGO, Rechia S, Freitas CMSM. Espaços públicos de lazer na promoção da qualidade de vida: uma revisão integrativa. LICERE-Revista do Programa de Pós-graduação Interdisciplinar em Estudos do Lazer. 2013;16(2)1-18. Disponível em: https://seer.ufmg.br/index.php/licere/article/view/372/267

14. Vasconcelos VJ, Rosa HC. Qualidade de vida a prática de atividades físicas no horto florestal em rio branco-ac. South American Journal of Basic Education, Technical and Technological 2016;3(1)137-141. Disponível em: http://revistas.ufac.br:8081/revista/index.php/SAJEBTT/article/view/514/306.

15. Szeremeta B, Zannin PHT. A importância dos parques urbanos e áreas verdes na promoção da qualidade de vida em cidades. RA'E GA - O Espaço Geográfico em Análise. 2013;29:177-193. Disponível em: DOI: http://dx.doi.org/10.5380/raega.v29i0.30747.

16. Castañon JAB, Paiva CR, Fonseca KM, Carneiro RS. Academias ao Ar Livre: uma análise dos espaços públicos. Blucher Engineering Proceedings. 2016;3(3):126-137. DOI: http://dx.doi.org/10.5151/engpro-conaerg2016-7011, https://doi.org/10.5151/engproconaerg2016-7011.

17. Silva DAS, Petroski EL, Reis RS. Barreiras e facilitadores de atividades físicas em frequentadores de parques públicos. Motriz. Rio Claro. 2009;15(2):219-227. Disponível em: http://bases.bireme.br/cgi-

bin/wxislind.exe/iah/online/?IsisScript=iah/iah.xis\&src=google\&base=LILACS\&lang=p\&nextAction= Ink\&exprSearch=535225\&indexSearch=ID.

18. Silva, C. L.. A atuação do profissional de Educação física: políticas públicas de esporte e lazer no contexto brasileiro. LICERE-Revista do Programa de Pós-graduação Interdisciplinar em Estudos do Lazer. 2014:17(1)28-35. Disponível em: https://www.ufmg.br/prpq/images/revistalicere/licerev17n01 ar1.pdf.

19. Scabar TG, Pelicioni AF, Pelicioni, MCF. Atuação do profissional de Educação Física no Sistema Único de Saúde: uma análise a partir da Política Nacional de Promoção da Saúde e das Diretrizes do Núcleo de Apoio à Saúde da Família-NASF. J Health Sci Inst. 2012; 30(4):411-8. Disponível em: http://www.unip.br/comunicacao/publicacoes/ics/edicoes/2012/04 outdez/V30 n4 2012 p411a418.pdf.

20. Giolo C, Ribeiro T, Pacheco RTB, Rodrigues JP. Programas públicos de atividade física e lazer em espaços não formais de educação: comparando as experiências das cidades de Valinhos/SP e Jundiaí/SP. Revista Brasileira de Estudos do Lazer. 2016;2(3):83-100. Disponível em: https://seer.ufmg.br/index.php/rbel/article/view/1925 .

21. Arana ARA, Xavier FB. Qualidade Ambiental e Promoção de Saúde: Um Estudo Sobre o Parque do Povo de Presidente Prudente-SP. Revista do Departamento de Geografia. 2016;2:1-14. https://doi.org/10.11606/rdg.v32i0.112038. 